\title{
Evaluation of iNSiGHT VET DXA (Dual- Energy X-ray Absorptiometry) for assessing body composition in obese rats fed with high fat diet: a follow-up study of diet induced obesity model for 8 weeks
}

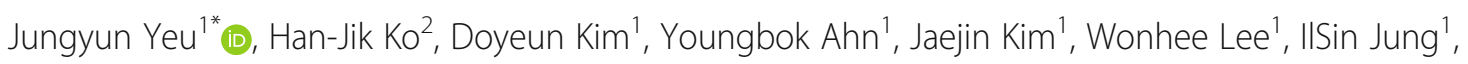
Jungkyu Suh ${ }^{2}$ and Seok-Jong Lee ${ }^{2}$

\begin{abstract}
We examined the precision, accuracy, and capability of detecting changes of Dual-Energy X-ray Absorptiometry (DXA) for the measurements of total-body weight (TBW), total-body fat weight (TBFW), and total-body lean weight (TBLW) in an 8-week follow-up study of rats. Twenty male rats (4-week) were divided into 2 diet groups. For 8 weeks, we measured body composition (TBW, TBFW, TBLW) by DXA and TBW by an electronic scale once a week. In week 8, we measured body composition 5 times by DXA and TBFW by dissecting experiment (EXP) of euthanized rats (12-week). Total-body fat ratio (TBFR) was defined as TBFW/(TBFW+TBLW). The precision of DXA was evaluated by measuring the coefficient of variation (CV) and accuracy was evaluated by comparing DXA-derived data with EXP data. The capability of detecting changes of DXA in follow-up study was verified by analyzing the trend of DXA-derived values over the 8 weeks. For TBW, TBFW, TBLW of DXA, CVs were $0.02 \pm 0.01,0.10 \pm 0.05,0.03 \pm 0.02$ and errors were $-6.996 \pm 3.429(r=0.999),+14.729 \pm$ $3.663(r=0.982),-21.725 \pm 4.223(r=0.991)$, respectively. Prediction models were [EXP TBW $=-31.767+1.085$ (DXA TBW), $\mathrm{R} 2=0.998$, root mean square error $(\mathrm{RMSE})=1.842]$ and [EXP TBFR $=-0.056+1.177$ (DXA TBFR), $\mathrm{R} 2=0.948, \mathrm{RMSE}=0.007]$. Over 8 weeks, DXA TBW and DXA TBLW steadily increased, DXA TBFW steadily increased followed by saturation or declination, difference of DXA TBFW between 2 diet groups steadily increased. In conclusion, our study verified that DXA (iNSiGHT VET DXA, OsteoSys, Korea) is accurate and precise enough to measure body composition of rats. Additionally, we confirmed the possibility that DXA could be used for the long-term follow-up studies.
\end{abstract}

Keywords: DXA, Body composition, Fat weight, Lean weight, Rat, Follow-up study

\footnotetext{
* Correspondence: jy_yeu@osteosys.com

'Osteosys, Seoul, Korea

Full list of author information is available at the end of the article
}

(c) The Author(s). 2019 Open Access This article is distributed under the terms of the Creative Commons Attribution 4.0 International License (http://creativecommons.org/licenses/by/4.0/), which permits unrestricted use, distribution, and reproduction in any medium, provided you give appropriate credit to the original author(s) and the source, provide a link to the Creative Commons license, and indicate if changes were made. The Creative Commons Public Domain Dedication waiver (http://creativecommons.org/publicdomain/zero/1.0/) applies to the data made available in this article, unless otherwise stated. 


\section{Introduction}

The World Health Organization (WHO) has acknowledged obesity as one of the most obvious public health problems, and the prevalence of obesity has almost doubled globally in 2008 compared to 1980. Obesity causes many health-related problems either independently or in association with other diseases; particularly with type 2 diabetes, cardiovascular disease, the upward trend of certain cancers, respiratory complications, and arthritis. Therefore, obesity shortens life span associating with an increased diseases risk, and it was reported that obesity shortens almost 7 years of life span for 40 years old population [1].

Studies preparing or treating obesity are actively in progress including clinical trials mainly conducted on mice and rats to analyze the effect of food, medications, and exercise on obesity. In most of these studies, the degree of increase in actual fat weight was estimated with the fat tissue extracted by dissecting experiment [2-5]. Dissection experiment (EXP) method of a euthanized animal can be relatively accurate compared with the noninvasive methods. However, EXP method is a limited approach as it cannot estimate the longitudinal change of the fat weight while continuously observing the growth of live animal.

Dual-energy X-ray Absorptiometry (DXA) instrument can be applied as an option for longitudinal observations of body composition without killing animals. DXA is considered to be a gold standard for measuring bone density and body composition in the human body [6], and studies are being conducted to utilize it in the field of animal experiments as well [7-9].

In this study, we conducted an 8-week follow-up study using iNSiGHT VET DXA (OsteoSys, Korea) on the diet-induced obese rats fed with a high-fat diet and the rats fed with a normal diet observing the change of total body weight, fat weight, and lean weight. The accuracy of total body weight was verified with an electronic scale that was used weekly alongside with DXA. After the feeding period, the main fat tissues of the euthanized rats were extracted to verify the accuracy of DXAderived fat weight by comparing the weights of the extracted fat tissues with the DXA-derived values. In addition, the data of five-time DXA scans without repositioning were analyzed for each rat to verify the basic precision of the DXA instrument.

\section{Methods/experimental}

\section{Animals}

A total of 20 male Sprague Dawley rats were the subjects of the study. At the beginning of the study, all rats were 4 week's old and divided into two groups of 10 rats. Each group was fed with a high-fat diet (HFD, 45\% kcal high-fat diet) and with a normal diet (ND, + 40 RMM-SP-10 Irradiated complete diet) respectively for the 8-week period.
The temperature of the animal room was maintained at $22 \pm 2{ }^{\circ} \mathrm{C}$, the relative humidity was $50.0 \pm 15.0 \%$, the illumination time was $12 \mathrm{~h} /$ day, the illumination was $150 \sim 300$ LUX, and the ventilation frequency was $10 \sim 20$ times / hour.

All process was carried out in accordance with the internal regulations of the animal ethics committee in Woojung Bio. IACUC approval number of animal experiment for this paper is WJIACUC20180731-3-11.

\section{Procedure}

During the feeding period, Total body Weight (TBW), Total Body Bone Weight (TBBW), Total Body Fat Ratio (TBFR), Total Body Fat Weight (TBFW) and Total Body Lean Weight (TBLW) were measured weekly by cone beam flat panel detector DXA (iNSiGHT VET DXA, Osteosys, Korea), and TBW was measured with an electronic scale (Entris, sartorius, Germany).

After 8 weeks of feeding, CO2 gas was used for euthanasia, and then all rats was scanned by DXA instrument five times without repositioning (1-min rest between measurements) and their TBW was measured with an electronic scale. The electronic scale was also used to measure the weight of the main fat tissues that were gained by dissecting experiment (EXP). The main fat tissues were extracted from subcutaneous fat, epididymal adipose tissue, kidney adipose tissue, and intestine adipose tissue. The EXP TBFW was calculated by $[$ EXP TBFW = sum of the weight of the extracted major fat tissues]. Since the TBBW was not measured separately in the dissection, it was also assumed that the DXA-derived TBBW was a true value. Therefore, the EXP TBLW was determined by [EXP TBLW $=($ EXP TBW - EXP TBFW) - DXA TBBW].

Until week 7, DXA measurements were performed on the rats that were sedated with gas inhalation (isoflurane) without supplemental anesthesia. Sedated rats were then placed on the scanning table with the prostrate position. DXA measurements, including $10 \mathrm{~s}$ of radiation output, took about $25 \mathrm{~s}$ per measurement. With the software provided by the manufacturer, bone areas were excluded from the collected X-ray images (Fig. 1), and then the body composition values were calculated by the embedded DXA algorithm.

To verify the accuracy, we calculated the differences and correlations between DXA-derived values and those obtained by dissecting experiment (EXP) on the euthanized rats. And then, the mean values and standard deviation of the errors of TBW, TBFW and TBLW were collected in all rats and the correlation values between the two measurement data were confirmed. In addition, a linear equation model predicting EXP values based on DXA-derived values were determined, and the R2 value was calculated. 


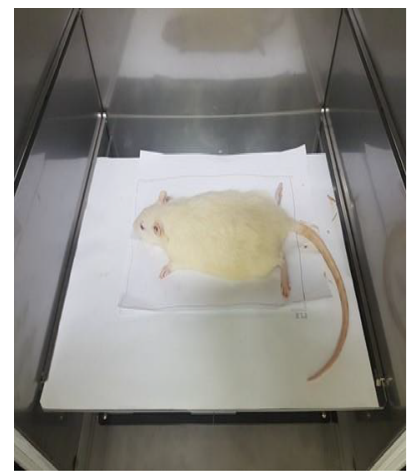

(a)

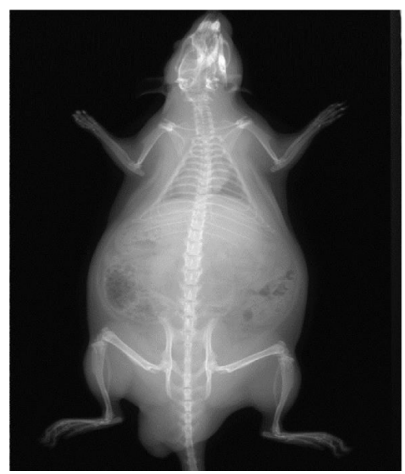

(b)

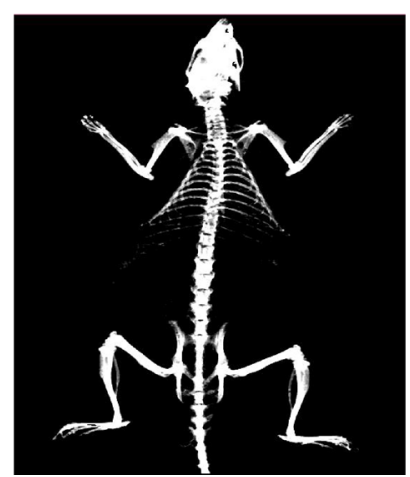

(c)

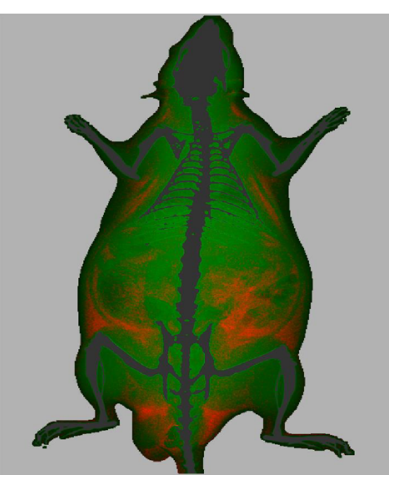

(d)

Fig. 1 A sample rat and its image. a A rat placed on the scanning table of the instrument (dual x-ray absorptiometry; DXA), b x-ray image, $\mathbf{c}$ bone enhanced image, $\mathbf{d}$ color composition image

The possibility of follow-up studies using DXA equipment was examined by comparing TBW and DXA TBW in regard to the mean value of all rats in the period of the 8 weeks. It was also examined whether the characteristics of each group changed consistently according to the intended experimental design by observing the changes of the mean TBFW of obesity-induced group and TBFW of the control group.

To verify the performance of DXA equipment, two quality control phantoms supplied with the instrument were measured weekly to collect fat ratio before a clinical test.

\section{Results}

The precision of the DXA instrument was verified by the coefficients of variation $(\mathrm{CV})$ of the repeated DXA analysis in Week 8. The mean coefficient of variation was lowest for DXA TBW of $0.02 \%$ and the highest for DXA TBFW of $0.10 \%$ (Table 1 ). The weekly change for fat ratio of two quality control phantoms (QP-ANI-1, QP-ANI-2) were 0.54 and $0.99 \%$ respectively for $\mathrm{CV}$ (Table 2).

The accuracy of the DXA instrument was first verified by comparing the data from dissecting experiment and those from DXA measurement in week 8(Table 3) and
(Fig. 2). Compared to the EXP values, DXA TBW was underestimated $(-6.996 \pm 3.429, r=0.999)$, DXA TBFW was overestimated $(14.729 \pm 3.663, r=0.982)$ and DXA TBLW was underestimated $(-21.725 \pm 4.223, r=0.991)$. In short, the DXA instrument estimated $98 \%$ of EXP TBW, 137\% of EXP TBFW, and 95\% of EXP TBLW.

It was confirmed that all EXP values and DXA-derived values (DXA TBW, DXA TBFW, and DXA TBLW) were highly correlated of R2 > 0.95 (Fig. 3), and based on this, we derived a regression equations for predicting EXP values from DXA-derived values (Table 4). EXP TBW can be predicted by DXA TBW [EXP TBW $=-31.767+1.085$ (DXA TBW), model R2 $=0.998$, root mean square error (RMSE) $=1.842 \mathrm{~g}]$. EXP TBLW and EXP TBFW were predicted by $[$ EXP TBLW $=($ EXP TBW - EXP TBBW $) \times(1-$ EXP TBFR)], and [EXP TBFW $=($ EXP TBW - EXP TBBW $)]$ respectively. As EXP TBBW were not measured by dissection, we assumed EXP TBBW as [EXP TBBW $=($ DXA TBBW)]. Therefore, EXP TBFR can be estimated from DXA TBFR by [EXP TBFR $=-0.056+1.177$ (DXA TBFR), model R2 $=0.948$, root mean square error $(\mathrm{RMSE})=0.007$ ]

The accuracy of the average DXA TBW for a total of 8 weeks was weekly verified by the average TBW measured with an electronic scale (Table 5) and (Fig. 4). Compared to TBW, DXA TBW was underestimated $(-4.951 \pm 5.008$,
Table 1 Basic precision of DXA values. The short-term precision of Dual-Energy X-ray Absorptiometry (DXA) calculated values in week 8 ( $n=20$ rats)

\begin{tabular}{ll}
\hline Contents & $\mathrm{CV}(\%)^{\mathrm{a}}$ \\
\hline DXA TBW $(\mathrm{g})$ & $0.02 \pm 0.01(0.01-0.04)$ \\
DXA TBFW (g) & $0.10 \pm 0.05(0.03-0.18)$ \\
DXA TBLW (g) & $0.03 \pm 0.02(0.01-0.06)$ \\
\hline
\end{tabular}

${ }^{a}$ The coefficient of variation are shown as mean \pm SD with the range shown in parentheses
Table 2 Precision of DXA values for fat content of phantom. The precision of fat percentage (fat \%) of quality control phantom measured by Dual-Energy X-ray Absorptiometry (DXA) for 8 weeks

\begin{tabular}{ll}
\hline Contents & $\mathrm{CV}(\%)^{\mathrm{a}}$ \\
\hline Fat \% of QP-ANI-1 (\%) & $0.54(30.51 \pm 0.17)$ \\
Fat \% of QP-ANI-2 (\%) & $0.99(32.05 \pm 0.32)$
\end{tabular}

${ }^{a}$ The coefficient of variation are shown as mean with the mean \pm SD of fat $\%$ shown in parentheses 
Table 3 Accuracy of DXA values in week 8. The accuracy and Pearson correlations of DXA-derived composition values of rats in week 8

\begin{tabular}{lllll}
\hline Contents & DXA $^{a}$ & EXP $^{b}$ & (DXA - EXP) $^{c}$ & Correlation coefficient $^{d}$ \\
\hline TBW (g) & $458.205 \pm 34.542(401.879-506.719)$ & $465.2 \pm 37.507(405-517.1)$ & $-6.996 \pm 3.429(-13.842--1.676)$ & $0.999, p<0.001$ \\
TBFW (g) & $54.019 \pm 14.324(33.975-94.748)$ & $39.29 \pm 16.445(19.722-87.955)$ & $14.729 \pm 3.663(6.794-20.749)$ & $0.982, p<0.002$ \\
TBLW (g) & $395.128 \pm 24.603(351.035-426.637)$ & $416.852 \pm 26.984(370.567-452.873)$ & $-21.725 \pm 4.223(-30.317--14.927)$ & $0.991, p<0.001$ \\
TBBW (g) & $9.059 \pm 0.858(7.723-10.169)$ & & &
\end{tabular}

${ }^{a}$ The DXA-derived values are shown as mean \pm SD with the range shown in parentheses

${ }^{\mathrm{b}}$ The EXP values are shown as mean \pm SD with the range shown in parentheses

'The errors of DXA-derived value from EXP value are shown as mean \pm SD with the range shown in parentheses

${ }^{d}$ The Pearson correlation coefficient value for DXA-derived values and EXP values calculated using "t-Test: Paired Two Sample for Means" in Excel

$\mathrm{r}=0.999)$. During the study period, the rats were divided into high-fat diet (HFD) group and normal diet (ND) group, and the changes of DXA-derived values (DXA TBW, DXA TBFW, and DXA TBLW) of these two groups were confirmed (Fig. 5). DXA TBW and DXA TBLW increased steadily in both groups from the beginning (week 0) to the end of the study (week 8). DXA TBFW increased steadily followed by saturation or declination at week 6 in both groups. However, the difference of DXA TBFW between the two groups showed increasing tendency until week 8 .

\section{Discussion}

\section{Precision}

The precision of DXA instrument can be affected by several factors. First, it is possible that a variation of the values results from the movement when the subject is breathing under anesthesia, or from the changed position and posture when the subject is placed on the scanning table of the instrument. The electrical noise of hardware such as X-ray tubes and detectors included in DXA instrument and the variation caused by errors in image processing or calculation algorithms for values in software also can affect the precision. In addition, environmental factors such as temperature and humidity, and changed hardware property can be considered as a variation factor affecting the study under a long period of time.
The precision of the DXA instrument to be verified in this study was for the short-term repeated measurement at the same position for each subject. We assumed that this was the most basic variation factors be confirmed before considering the effect of the movement or of the changed position/posture by the subject. Especially, if the precision of the short-term repeated measurement is high, precise results can be obtained without additional measuring of the same subject nor increasing the measuring time.

The coefficient of variation (CV) calculated from the sample standard deviation $(n=5)$ of DXA TBW, DXA TBFW, and DXA TBLW after euthanasia was highest in average of DXA TBFW $(n=20)$ of $0.10 \%$, and the maximum value was $0.18 \%$ (Table 1 ). Considering the average DXA TBFW which was $54.019 \mathrm{~g}$ (Table 3), the standard deviation between the short-term repeated measurements of the DXA instrument used in this study could be verified with significantly high precision to the degree of $0.054 \mathrm{~g}$ from the DXA TBFW value.

In addition, the precision of long-term repeated measurements for the DXA instrument was also verified. For this purpose, the quality control phantoms provided with the DXA instrument was measured once a week before the test to check the range of fat percentage (fat $\%$ ) and CV (Table 2). The reason for applying fat percentage corresponding to TBFR, instead of directly verifying TBW, TBFW, or TBLW, was because there was

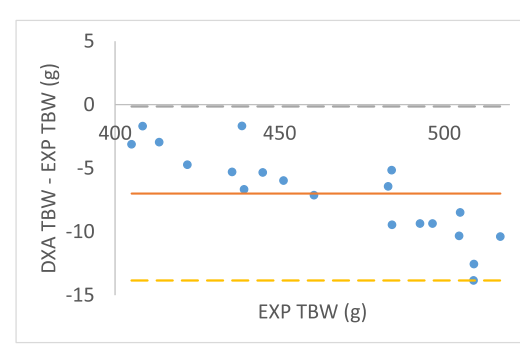

(a)

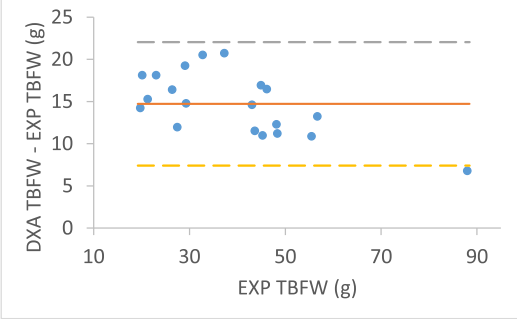

(b)

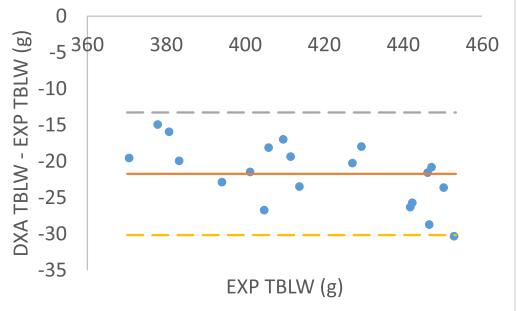

(c)

Fig. 2 Residual plots of DXA values in week 8. Residual plots of DXA-derived values in week 8 for a total body weight (TBW), b total body fat weight (TBFW), and $\mathbf{c}$ total body lean weight (TBLW). The filled circles show the errors with dissecting experiment (EXP) for each rats with the solid line being the mean difference. The large dashed lines represent the mean \pm 2 SD 


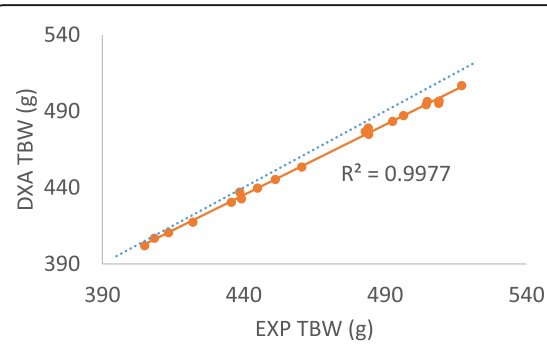

(a)

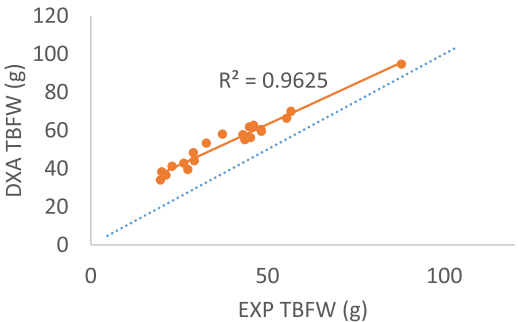

(b)

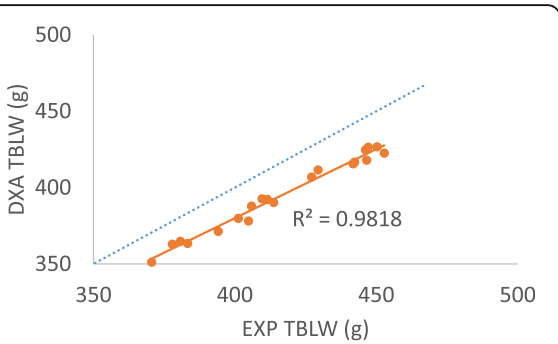

(c)

Fig. 3 Relationship between DXA and EXP values in week 8. Scatter plots of the relationship of the values between DXA and EXP in week 8 for $\mathbf{a}$ TBW, $\mathbf{b}$ TBFW, and $\mathbf{c}$ TBLW. The small dashed lines represent the point where the values are same. The solid lines represent the trends of their relationship with $\mathrm{R}$-squared values

the difference between the weight of phantom and those of the rats. As the fat percentage ranges from 0 to $100 \%$ regardless of the weight of the individual, it can be used to estimate the variation in DXA TBFW in the rats resulted from the changed hardware property over longterm repeated measurements.

For the two quality control phantoms, the $\mathrm{CV}$ of fat percentage calculated from the weekly measured data for 8 weeks was 0.54 and $0.99 \%$. That is, the precision of the fat percentage was controlled within $1 \% \mathrm{CV}$ during the test. In the QP-ANI-2 phantom, the mean value of fat percentage was $32.05 \%$ and the standard deviation was $0.32 \%$. Considering the average weight of the rats (DXA TBW - DXA TBBW) which was $449.146 \mathrm{~g}$ (Table 3), it could be estimated that the standard deviation of DXA TBFW or DXA TBLW resulted from the changed hardware property over the test period was $1.437 \mathrm{~g}$ in maximum.

\section{Accuracy}

To verify the accuracy, it is mandatory to have a reference value for comparison. In this study, the main fat tissue was extracted through a dissecting experiment (EXP) to verify the accuracy of the body composition of the rats measured by the DXA instrument. We assumed the sum of the weight of fat tissues measured by the

Table 4 Prediction equations for EXP values. The prediction equation for the dissecting experiment (EXP) values from DualEnergy X-ray Absorptiometry (DXA) derived composition values in week 8

\begin{tabular}{llc}
\hline Contents & Regression equations $^{\mathrm{a}}$ & Model R2 $^{\mathrm{b}}$ \\
\hline EXP TBW $(\mathrm{g})$ & $-31.767+1.085$ (DXA TBW) & 0.998 \\
EXP TBFR (\%) & $-0.056+1.177$ (DXA TBFR) & 0.948 \\
EXP TBBW $(g)$ & DXA TBBW & \multicolumn{2}{c}{} \\
\hline
\end{tabular}

a"Least squares" method was used

'The coefficient of determination for estimating regression equation

'This model for 'EXP TBBW' is the assumption electronic scale as the total fat weight (TBFW). We also measured the total weight (EXP TBW) of the rats prior to dissection and assumed that DXA TBBW was true because the bone mineral contents corresponding to EXP TBBW were not measured. Using these values, the lean weight (EXP TBLW) of the rats was calculated and used as a reference value. All rats $(n=20)$ were euthanized after 8 weeks of follow-up and then dissections were performed. The values measured from dissections were compared with the DXA-derived values to verify the accuracy of the DXA instrument.

DXA TBW was underestimated with the error of $-6.996 \pm 3.429 \mathrm{~g}$ compared to EXP TBW (Table 3). It was lower about $1.53 \%$ than the average weight of $458.205 \mathrm{~g}$, which seemed to be affected by the fact that the tail was not entirely included in the measurement image of the rat, rather than the error of the DXA instrument. The imaging area of this DXA instrument was sufficient to include the entire body of the rat, but part of the tail was not included in the image when the posture of the rat was fully prostrated (Fig. 1). Given this point, we could conclude that the weight estimating performance of this DXA instrument for the measurable areas was even more accurate than the obtained results.

DXA TBFW and DXA TBLW were estimated by DXA software from DXA TBW, DXA TBBW and DXA TBFR. DXA TBFW was overestimated with the error of $14.729 \pm 3.663 \mathrm{~g}$ compared to EXP TBFW and DXA TBLW underestimated with the error of $-21.725 \pm 4.223 \mathrm{~g}$. In other words, there was a difference between the DXA TBFR and the EXP TBFR. The algorithm in the DXA software might have caused the difference, but the fat tissues located other than the ones extracted from the dissection could be regarded as the possible reason. For more accurate measurement and verification for fat mass, there was the chemical analysis called Carcass method [9], but we excluded this method in this study from the perspective of time, process, and budget requested for the analysis of the method. 
Table 5 Accuracy of DXA values for 8 weeks. The accuracy and Pearson correlation of Dual-Energy X-ray Absorptiometry (DXA) derived composition values with the electronic scale values of rats for 8 weeks

\begin{tabular}{|c|c|c|c|c|}
\hline & $\mathrm{DXA}^{a}$ & Electronic scale ${ }^{b}$ & $(\text { DXA - Electronic scale) })^{c}$ & Correlation coefficient $^{\mathrm{d}}$ \\
\hline TBW (g) & $324.248 \pm 111.581(134.508-458.205)$ & $329.198 \pm 116.164(132.945-465.2)$ & $-4.951 \pm 5.008(-12.312-1.563)$ & $0.999, p<0.009$ \\
\hline
\end{tabular}

Due to the strong correlation $(\mathrm{R} 2>0.95)$ (Fig. 3), it was suggested that this DXA instrument was accurate enough to predict the EXP values while there were errors between EXP values and DXA-derived values in TBFW and TBLW. Therefore, we derived a simple linear equation to predict the EXP values using DXA-derived values. As the ratio of DXA TBFW to DXA TBLW determines the DXA TBFR, all values can be estimated by DXA TBFR. Therefore, we only estimated the relationship between EXP TBW and EXP TBFR and DXAderived values. The $\mathrm{R} 2$ values of the derived equation for EXP TBW was 0.998 and for EXP TBFR was 0.948 . Because R2 from both predictions were higher than 0.9 , the equations by DXA measurements would be a simple substitute if the similar dissections are requested in the other experiment.

The another purpose of this study was to evaluate whether the difference in body composition of high-fat diet (HFD) group and that of normal diet (ND) group can be accurately estimated by DXA instrument.

Because of the impossibility to conduct the dissections during the 8-weeks feeding period, it was TBW by the electronic scale for each rat that could be collected only during the 8-week period to be compared directly with DXA-derived values. However, by comparing the TBW and the DXA TBW, it was confirmed that the weight of the rats were estimated very accurately as the result of dissection in week 8 . The average of error of the DXA TBW $(n=20)$ was $-4.951 \pm 5.008 \mathrm{~g}$ compared to the
TBW (Table 5), and the accuracy of estimating the actual weighing scale with DXA TBW was $\mathrm{R} 2=0.999$ (Fig. 4).

Analysis of the results from the dissection in week 8, it was confirmed that DXA-derived values (TBW, TBFW, and TBLW) estimated with high accuracy, and also confirmed that weekly measured DXA TBW until week 8 was accurate. Therefore, we could estimate that DXA TBFW and DXA TBLW until week 7 would be reliable enough as well.

Another context in support of this estimation was confirmed by dividing DXA-derived values into the HFD Group and the ND Group (Fig. 5). The difference between the two groups in the DXA TBFW showed a steady increase over the test. Finally, in the last week 8 , we found a similar difference between the two groups in the TBFW and the TBFW even though TBFW was measured with lower values compared to TBLW. The TBFW difference between the two groups was significantly larger $(20.50 \mathrm{~g}$ at week 8$)$ than the estimated variation $(1.450 \mathrm{~g})$ by the changed condition of the instrument during the test period. Therefore, it was possible to confirm that the high-fat diet has a clear effect on the fat gaining process. TBW and TBLW continued to increase while TBFW decreased in both groups from week 6 . Nonetheless, the increasing tendency in the differences between the two groups supported the assumption that the DXA instrument would be able to estimate even small changes in the rat.

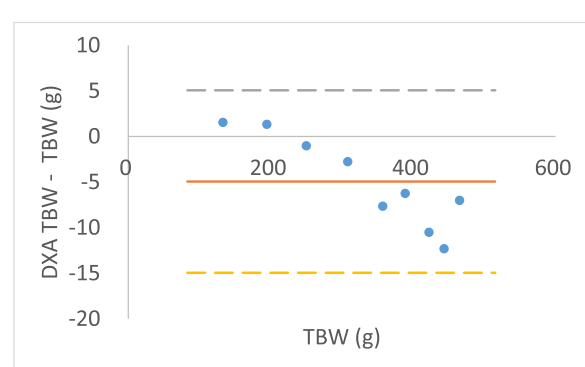

(a)

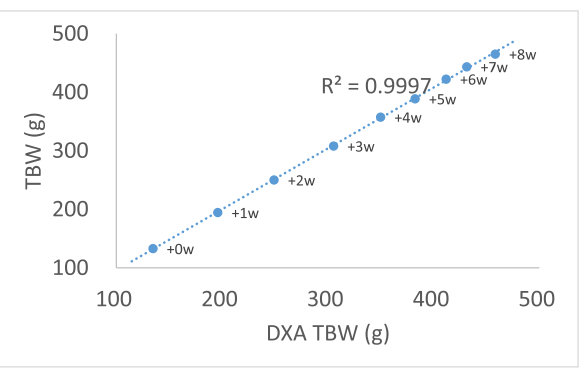

(b)

Fig. 4 Residual plot and relationship between DXA and EXP values for 8 weeks. a Residual plot of DXA-derived TBW values for 8 weeks. Each filled circle show the mean error of all rats $(n=20)$ with the values measured by an electronic scale for each weeks with the solid line being the mean difference. The large dashed lines represent the mean \pm 2 SD. b Scatter plot of the relationship of mean TBW $(n=20)$ between DXA and an electronic scale for 8 weeks with the labels represented each weeks. The small dashed line represents the point where the values are same. R-squared value of their trend line is also represented in graph 


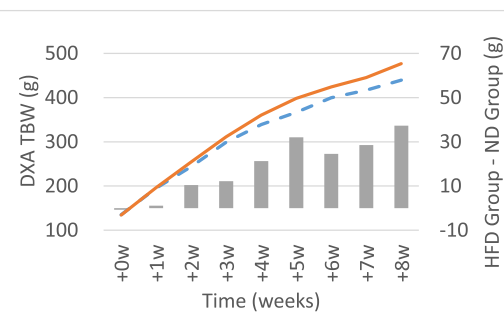

(a)

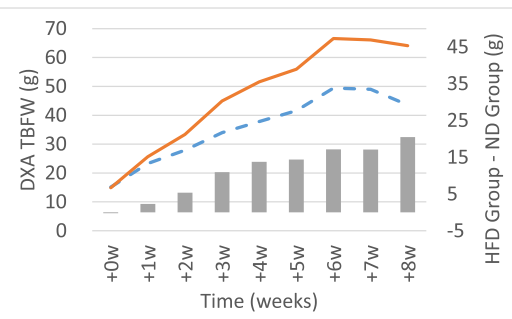

(b)

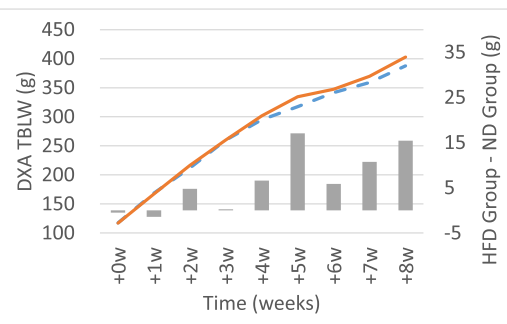

(c)

Fig. 5 Trend graph of DXA values for 8 weeks. The graphs represent the trend of mean DXA values divided into the high fat diet (HFD) group and the normal diet (ND) group for 8 weeks for (a) TBW, (b) TBFW, and (c) TBLW. The solid lines are the trend of HFD group and small dashed lines are the trend of ND Group. Bar graph means the difference between two groups

\section{Conclusions}

Based on the abovementioned review of the accuracy, we can suggest that follow-up test for measuring the change of fat weight and lean weight can be conducted without extra dissection if the test is prepared with the DXA instrument.

The limitation of this paper is that no direct validation of other body composition values of DXA except the TBW value was performed during the lifetime of the rat. Therefore, in order to overcome this limitation, it will be necessary to compare with other validated instruments or to perform weekly dissecting experiment with a sufficient number of animals.

\section{Abbreviations}

CV: Coefficient of Variation; DXA: Dual-Energy X-ray Absorptiometry; EXP: Dissecting Experiment; HFD: High Fat Diet; ND: Normal Diet; RMSE: Root Mean Square Error; TBBW: Total Body Bone Weight; TBFR: Total Body Fat Ratio; TBFW: Total Body Fat Weight; TBLW: Total Body Lean Weight; TBW: Total Body Weight; WHO: The World Health Organization

\section{Acknowledgements}

Not applicable.

\section{Funding}

Besides the support for measuring instrument and the official test fee, there was no separate funding from Osteosys to Woojung Bio. The method applied in this test was planned by Osteosys, and the purchase of rats, selecting diets appropriate for the conditions, dissection of the rats were conducted by Woojung Bio.

\section{Availability of data and materials}

Data will not be shared.

\section{Authors' contributions}

JY designed this experiment and managed the whole process. H-JK was responsible for animal breeding and dissection experiments. DK created a PC application for the experiment. YA was responsible for the final review and approval of the designed experiment. JK coordinated the situation between the two companies for the experiment and reviewed the suitability of the data collected during the experiment. WL advised on how to analyze the data and reviewed the contents of the paper. IJ collected the existing research related to this paper and conducted the English translation work. JS managed general aspects of animal experiments. S-JL served as a technical support for animal experiments and assisted in the detailed design of the experiments. All authors read and approved the final manuscript.

\section{Competing interests}

This study was conducted based on the results of clinical trial commissioned by Osteosys, a manufacturer of iNSiGHT VET DXA, to Woojung Bio.

\section{Author details}

${ }^{1}$ Osteosys, Seoul, Korea. ${ }^{2}$ Woojung Bio, Suwon, Korea.

Received: 17 March 2019 Accepted: 29 April 2019

Published online: 24 June 2019

\section{References}

1. Song DK, Sung YA. Obesity: introduction. Korean J Med. 2013;84(5):619-23.

2. Hong KH, Kim HS, Jang KH, Kang SA. The improvement effects of $\beta$-glucan on adiposity and serum lipids levels in high fat diet-induced obese rats. J Korea Academia-Industrial Coop Soc. 2015;16(6):3973-81.

3. Choi DH, Cho JY. The effect of low intensity exercise on expression of inflammatory response and apoptosis in rats with obesity induced by a high-fat diet. Exerc Sci. 2018;27(1):40-9.

4. Jeong HS. Efficacy of Alismatis Orientale Rhizoma on obesity induced by high fat diet. Korea J Herbol. 2013;28(3):95-106.

5. Go GY, Park JH, Kwak JY, Ahn TW. Anti-obesity effect of Yeoldahanso-tang on obesity mice. J Sasang Constitut Med. 2017;29(2):154-73.

6. Scafoglieri A, Clarys JP. Dual energy X-ray absorptiometry: gold standard for muscle mass? J Cachexia Sarcopenia Muscle. 2018;9(4):786-7.

7. Eleftheriades M, Vafaei H, Dontas I, Vaggos G, Marinou K, Pervanidou P, Sebire NJ, Chrousos GP, Nicolaides KH. Assessment of body composition in Wistar rat offspring by DXA in relation to prenatal and postnatal nutritional manipulation. Pediatr Res. 2016;80(2):319-25.

8. Sontam DM, Vickers MH, O'Sullivan JM, Watson M, Firth EC. Different shortterm mild exercise modalities Lead to differential effects on body composition in healthy Prepubertal male rats. Biomed Res Int. 2015;2015:404201.

9. Nagy TR, Clair AL. Precision and accuracy of dual-energy X-ray absorptiometry for determining in vivo body composition of mice. Obes Res. 2000;8(5):392-8.

\section{Publisher's Note}

Springer Nature remains neutral with regard to jurisdictional claims in published maps and institutional affiliations.

Ready to submit your research? Choose BMC and benefit from:

- fast, convenient online submission

- thorough peer review by experienced researchers in your field

- rapid publication on acceptance

- support for research data, including large and complex data types

- gold Open Access which fosters wider collaboration and increased citations

- maximum visibility for your research: over $100 \mathrm{M}$ website views per year

At BMC, research is always in progress.

Learn more biomedcentral.com/submission 